\title{
Mechanical properties of the thin-walled welded straws for the COMET experiment
}

\author{
Michail Kravchenko ${ }^{1}$ \\ Joint Institute for Nuclear Research \\ Joliot-Curie 6, Dubna, 141980, Russia \\ E-mail:kmd@jinr.ru

\begin{abstract}
Alexander Volkov
Joint Institute for Nuclear Research

Joliot-Curie 6, Dubna, 141980, Russia

E-mail:adv@jinr.ru
\end{abstract}

\section{Aliaksei Paulau \\ Joint Institute for Nuclear Research \\ Joliot-Curie 6, Dubna, 141980, Russia \\ E-mail:pavlov@jinr.ru}

The purpose of this work was to investigate the most important mechanical properties of the thinwalled welded straws developed by JINR (Joint Institute for Nuclear Research) COMET (COherent Muon to Electron Transition, J-PARK) group and to estimate straws lifetime as the main part of the COMET experiment tracking system. The results of the measurements, as well as the main parameters of the stand for studying the straw characteristics are given.

European Physical Society Conference on High Energy Physics - EPS-HEP2019 -

10-17 July, 2019

Ghent, Belgium

\section{${ }^{1}$ Speaker}




\section{Introduction}

COMET (COherent Muon to Electron Transition, J-PARC, Japan) is one of the experiments that study the extended theory of elementary particle physics. The main task of the COMET experiment is to search for physics beyond the SM through the detecting the process of neutrinoless conversion of muons into electrons in the field of a nucleus (muon conversion, $\mu-\mathrm{N}$ $\rightarrow \mathrm{e}^{-} \mathrm{N}$ ); a lepton flavor violating process. Phase-I will operate two different configurations. The primary COMET Phase-I detector for $\mu$-e conversion measurement is composed of a cylindrical drift chamber (CDC) and a set of hodoscope counters for triggering and timing, called the CyDet detector. The second configuration has an aim to make a direct measurement of potential background for the full COMET experiment using prototypes of the Phase-II straw tracker and the electron calorimeter (ECAL), called StrEcal detector. Phase-II will use the information gained in Phase-I, a much more intense beam and a more complex detection system to achieve a further two orders of magnitude of sensitivity.

\section{Test Bench}

To ensure high coordinate accuracy of the straw detector, precision positioning of the wire inside the tube and the tubes themselves in the detector modules is required. The material from which the straw is made is also required to preserve its basic physical properties in time, as well as to be homogeneous throughout the length. The most important physical properties of the straw material are the area of elastic deformation, the value of the elastic modulus, which characterizes the straw strength, the rate of stress relaxation. Knowledge of the Poisson's ratio is required to select the straw initial tension, since its tension changes when operating in a vacuum. Test bench (fig. 1) has a simple design that allows rebuilding, adding or removing parts and mechanisms for carrying out a wide range of straw characteristics measurements. End-caps of investigated straw are glued to its ends. One straw end cap is connected to a tension gauge rigidly fixed on the base of the bench. The other one is connected to a straw tension system using a screw. Stand's units are enclosed in a sealed volume formed by a metal base and a Plexiglas body. Only a thermostabilization system was used for all measurements, the temperature was $23^{\circ} \mathrm{C}$, unless otherwise specified. The measurements were carried out on welded straws of polyethylene terephthalate with a diameter of $9.8 \mathrm{~mm}$, a wall thickness of $20 \mu \mathrm{m}$ and one-sided of $70 \mathrm{~nm}$ thick aluminum. The film for the straw was produced by Ltd SPE "POLYPLEN" (Russia). The straws was produced and the bench was created by JINR COMET group.
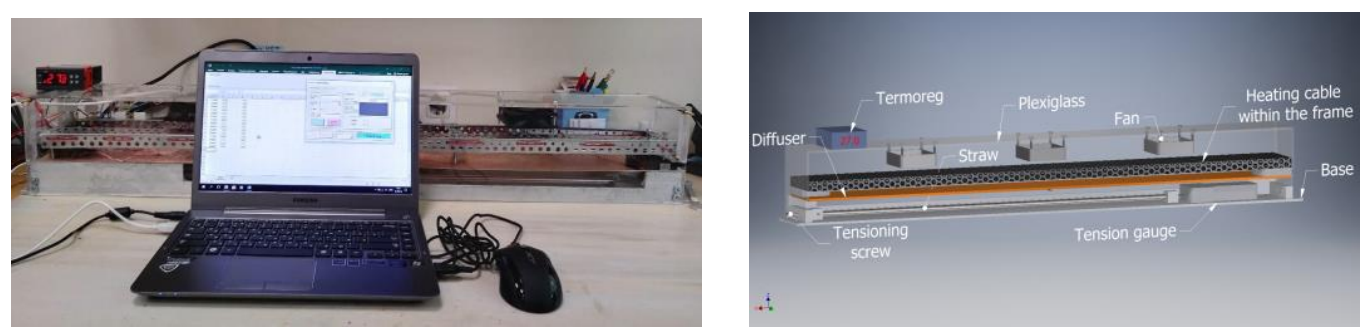

Fig. 1. The general and schematic view of the created test bench 


\section{The results of the measurements}

\subsection{Stress-strain dependence}

Up to the stress of $30 \cdot 106 \mathrm{~N} / \mathrm{m} 2$ (fig. 2) the dependence has linear character, deformation is elastic and submits to Hooke's law, which corresponds to a tension of $(1850 \pm 1)$ gf and serves as a boundary of the elastic stress region. Exceeding this tension value causes the appearance of a plastic component of deformation, which increases the rate of tension relaxation and straw creep, and dependence becomes nonlinear. Line 2 corresponds to elastic deformation only. The modulus of elasticity $\mathrm{E}$ is equal to the slope ratio of this dependence and in this case: $\mathrm{E}=(4.44 \pm 0.05) \cdot 109$ $\mathrm{N} / \mathrm{m} 2$. The Poisson's ratio was calculated: $0.338 \pm 0.004$.

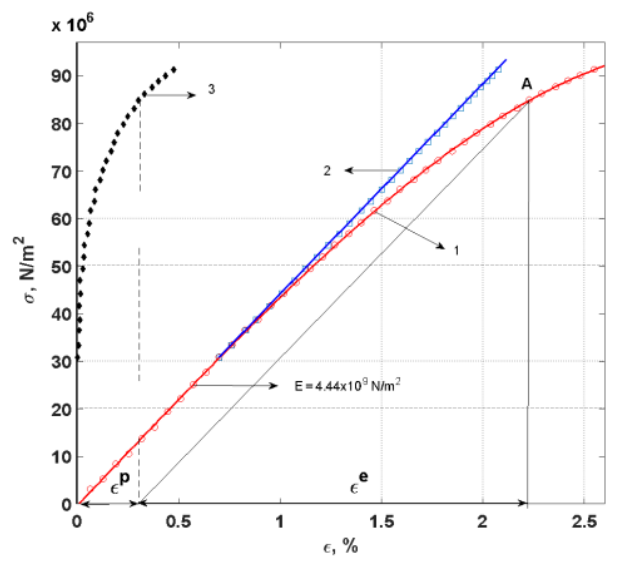

Fig. 2. Areas of elastic and plastic deformation of straw.

\subsection{The elastisicy modulus on temperature dependence}

From the obtained dependence follows that the modulus value in the temperature range (12 $-21)^{\circ} \mathrm{C}$ is larger (fig. 3), which causes lower straw deformation and creep. Thus in the experiment straw detector should operate in this range.

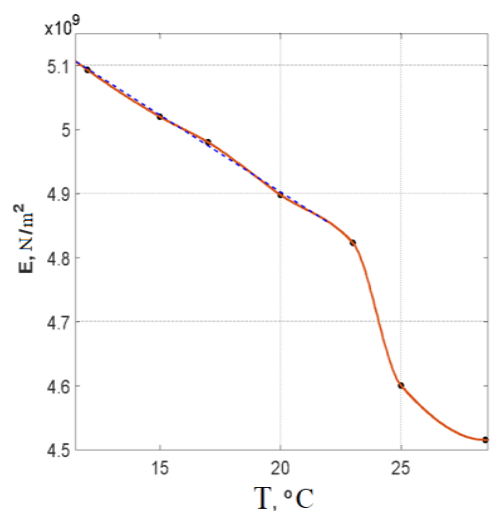

Fig. 3. The elastic modulus on the temperature dependence.

\subsection{The elasticity modulus on the straw thickness dependence}

The elasticity modulus decreases with increase in the straw thickness. This effect is associated with film production technology for a straw. The thin film production technology involves rolling a thicker film between the rollers with a smaller gap between them than the initial film thickness. This processes will cause a decrease in the straw longitudinal deformation during 
its tensioning which characterizes a higher value of the elastic modulus. A three times increase in film thickness resulted in a $32 \%$ decrease in modulus.

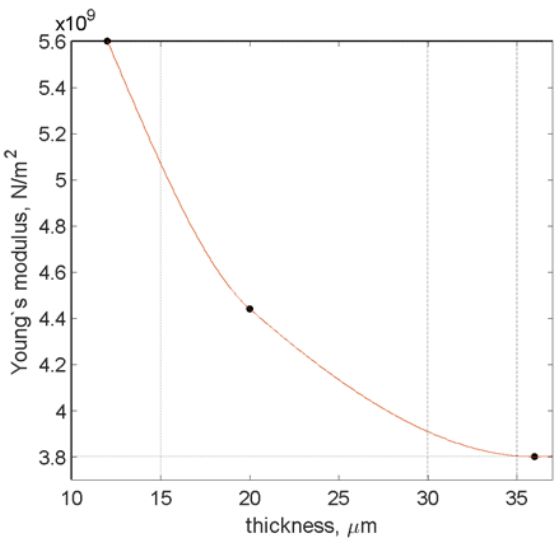

Fig. 4. The elastic modulus on the straw wall thickness dependence.

\subsection{The stress relaxation of the straw}

The stress relaxation of the straw (tension relaxation) is a process of decreasing the stress inside the material and its tension under constant length, i.e. constant total strain. Therefore, it is important to ensure the initial tension at which the cylindrical shape and the required accuracy of event registration remain for a long time. The temperature inside the test bench was $(+27 \pm 0.5)$ ${ }^{\circ} \mathrm{C}$. The fig. 5 presents data on stress relaxation for 290 days.

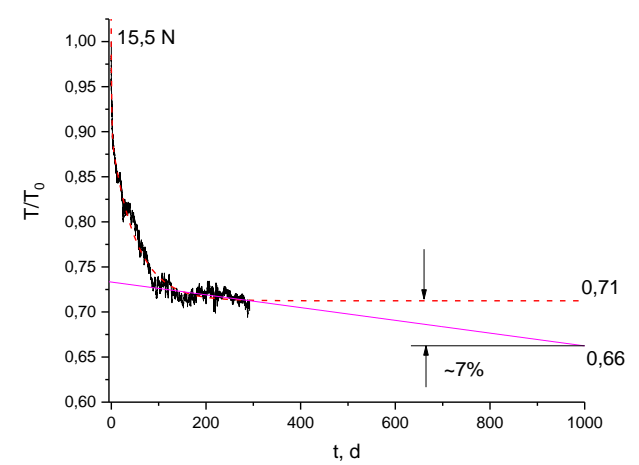

Fig. 5. The stress relaxation within 290 days.

The initial tension was $15.5 \mathrm{~N}$. There are two main processes of the relaxation: physical (represents fast component) and chemical relaxation (represent slowest one). In this regard, the double exponential function with a free term was selected for approximating and extrapolating the experimental data, because takes into account the fact of a sharp decreasing in the relaxation rate, starting from the 90th day, when the stage of fast relaxation component ends. For a more realistic estimate, a linear approximation of the data's tail (starting from the 90th day) was made. The main result is that after 3 years (COMET straw tracker lifetime) the straw tension will be about $66 \%$ of initial. Taking this into account the optimal initial straw tension can be determined. In the COMET case, it is $1.23 \mathrm{kgf}$.

\section{References}

[1] COMET Collaboration, COMET Phase-I Technical Design Report, arXiv:1812.09018;

[2] A. D. Volkov et al. The test bench to study the characteristic of straw tubes. //Advances in applied physics, 2019, V. 7, № 1, p. 76-84. 